\title{
Young patient's secondary prevention in ischemic stroke with the percutaneous closure of the patent foramen ovale and postoperative repermeabilisation - case report
}

\author{
Teodor-Valentin Stanciu ${ }^{1,2}$, Bianca Nemes ${ }^{1,2}$, Diana Mariesan ${ }^{1,2}$, \\ Vitalie Vacaras ${ }^{1,2}$, Dafin Fior Muresanu ${ }^{1,2}$ \\ ${ }^{1}$ Neuroscience Department, "Iuliu Hatieganu" University of Medicine and Pharmacy, Cluj-Napoca, Romania \\ ${ }^{2}$ County Clinical Emergency Hospital, 2nd Neurology Clinic, Cluj-Napoca, Romania
}

\begin{abstract}
Introduction. Cryptogenic stroke represents $25 \%$ of all cases. Paradoxical embolism is a common case in people under 45 years old who have patent foramen ovale.

Case presentation. 44-years old patient with left motor deficit, dysarthria and dizziness suddenly installed after physical effort. During neurological evaluation, the patient had a central type facial paresis, slight asymmetry of the palatine tonsil, left motor hemiparesis (4/5), dysmetria and dysarthria. Biological examinations showed an association with MTHFR and heterozygous $V$ factor mutation. Computed tomography was made brain with venous sequence where we could see hypoplastic right vertebral artery. MRI revealed a stroke in the territory of the superior cerebellar artery, bilaterally. Transesophageal ultrasound described a patent foramen ovale and an atrial septal defect. RoPE scale was 8 which meant a big risk for paradoxical embolism. Surgical correction was made for secondary prevention of stroke. After 3 months, the patient made a transcranial ultrasound with microbubbles contrast agent which detected a permeable right-to-left shunt.

Conclusions. The ischemic stroke and patent foramen ovale with paradoxical embolism mechanism are common in young adults. Postinterventional repermeabilization are rare cases which can raise many questions about prophylaxis and treatment.
\end{abstract}

Keywords: ischemic stroke, paradoxical embolism, patent foramen ovale

\section{Abbreviation}

MRI - magnetic resonance imaging

RoPE - risk of paradoxical embolism

ESUS - embolic stroke of undetermined source

PFO - patent foramen ovale

NIHSS score - National Institute of Health Stroke score

ASPECT score - Alberta stroke program early CT score

FT4 - free thyroxine

$\mathrm{TSH}$ - thyroid stimulating hormone

ATPO - anti-thyroid peroxidase

ATG - anti-thyroglobulin

AAN - antinuclear antibodies

\section{INTRODUCTION}

Cryptogenic ischemic stroke represents $25 \%$ of all stroke cases. They are cases with unknown em-
CIC - antibody immune complexes

p-ANCA - antineutrophil cytoplasmic antibodies

$\mathrm{RNP}$ - ribonucleoprotein

FLAIR - fluid-attenuated inversion recovery

DWI - diffusion weighted imaging

TTE - transthoracic ultrasound

HITs - high intensity transients

TCD - transcranial ultrasound

ASA - atrial septal aneurysm

CT - computed tomography bolic sources (ESUS). The origin can be: calcified mitral valve, paradoxical embolizations, atheroma plaques in the aortic artery or in the large cerebral arteries (arterial-arterial mechanism) (1). 
Patent foramen ovale (PFO) is an innate septal defect which normally closes in early periods. In $25 \%$ of people, PFO does not close. It is a problem when in the venous system we have a blood clot which can cross this innate septal defect and to migrate to the periphery (2-4). Nowadays, we can use many instruments to diagnose patent foramen ovale. Transesophageal ultrasound is the gold standard. Transcranial Doppler ultrasound with microbubbles have the same sensibility and specificity in right-to-left shunt detection. However, Valsalva maneuver is made easier in transcranial doppler ultrasound. So, it is much easier to evaluate right-to-left shunt in this way (5). In order to publish this case, the verbal consent of the patient was obtained.

\section{CASE PRESENTATION}

44-years old patient, nonsmoker, with hydrostatic varicose veins, without treatment who came in the emergency service with left motor deficit, dysarthria and dizziness suddenly installed in the morning. After an hour, the patient had an easy amelioration of neurological symptoms. We were informed that he had made physical effort a day before (cycling).

The neurological clinical examination, at the time of presentation, showed a left facial paresis of central type, with asymmetry of the palatine tonsil, with a deviation to the right of the palatine tonsil at the pronunciation of the vowel "a", associated with a difficulty swallowing solids, decreased muscle strength in the left limbs (4/5), indifferent plant in the left leg, dysmetria and dysarthria. NIHSS was 5 points.

The patient didn't have indication for thrombolytic treatment, because he was out of the therapeutic window.

\section{Paraclinical evaluations}

Brain computed tomography was normal (aspect score 10 points). Brain computed tomography with angiography exclude vertebral artery dissection. This examination showed just an hypoplastic right vertebral artery (figure 1).

Laboratory tests showed a slight folic acid deficiency - $171 \mathrm{pg} / \mathrm{ml}$ (normal value 180-914 pg/ml). Thyroid hormones (FT4, TSH, ATPO, ATG) and immunological markers (AAN, CIC, p-ANCA,

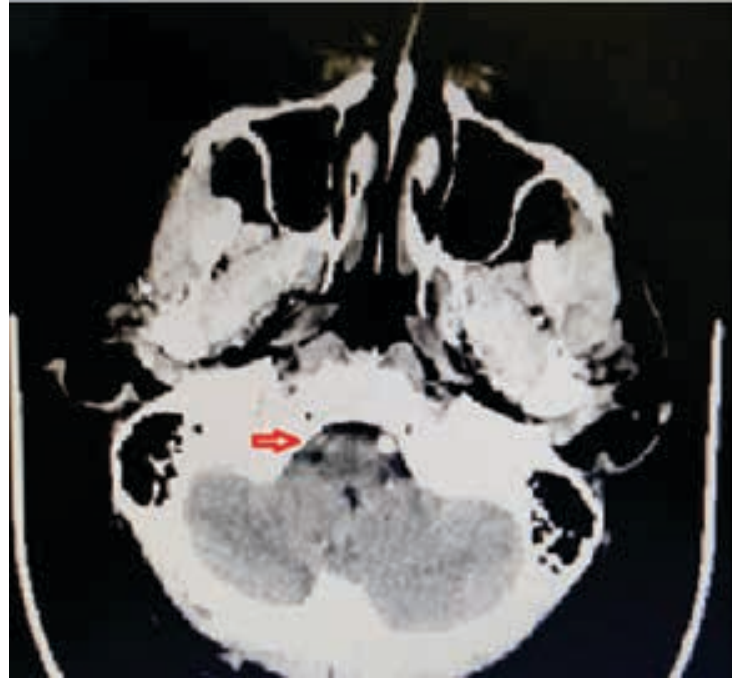

FIGURE 1. Brain computed tomography with angiography. Axial section. Hypoplastic right vertebral artery

antibodies RNP/SM/RO CCP) were normal. Because, thrombophilia has a high incidence at this age, thrombophilia profile was made which showed MTHFRc mutation and heterozygous $\mathrm{V}$ factor mutation.

To exclude a possible arterio-arterial embolism event we made a doppler ultrasound which was normal.

Brain magnetic resonance imaging describes a lot of ischemic areas in bilateral cerebellar emispheres and left parietal lobe, T1, T2, FLAIR hyperintensity and an increased DWI signal in the left cerebellar lobe. On the left we found a minimal thalamic lesion with T2 and FLAIR hyperintensity, without DWI signal, which corresponds with superior cerebellar artery area (figures 2-7).

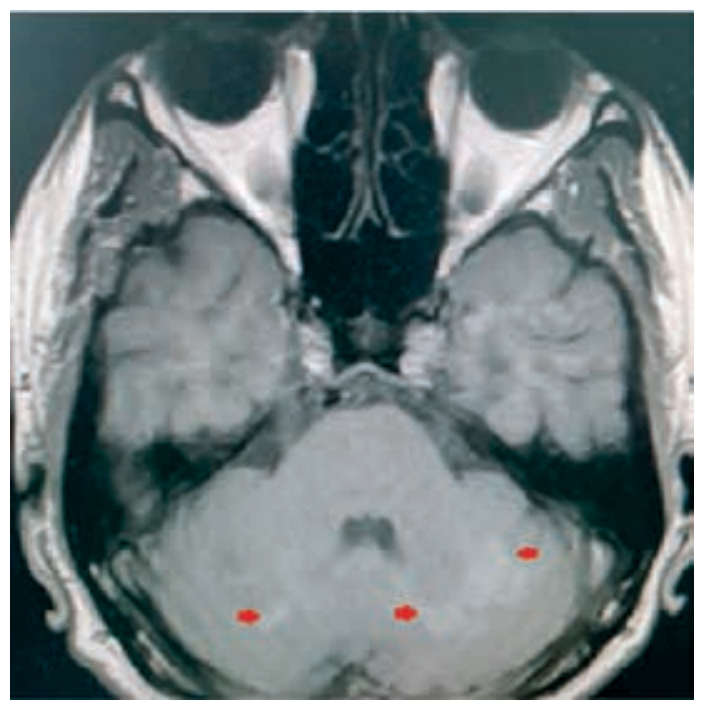

Figure 2. Brain magnetic resonance imaging. Axial section. T1. Minimal hyperintensity in bilateral cerebellar hemispheres 


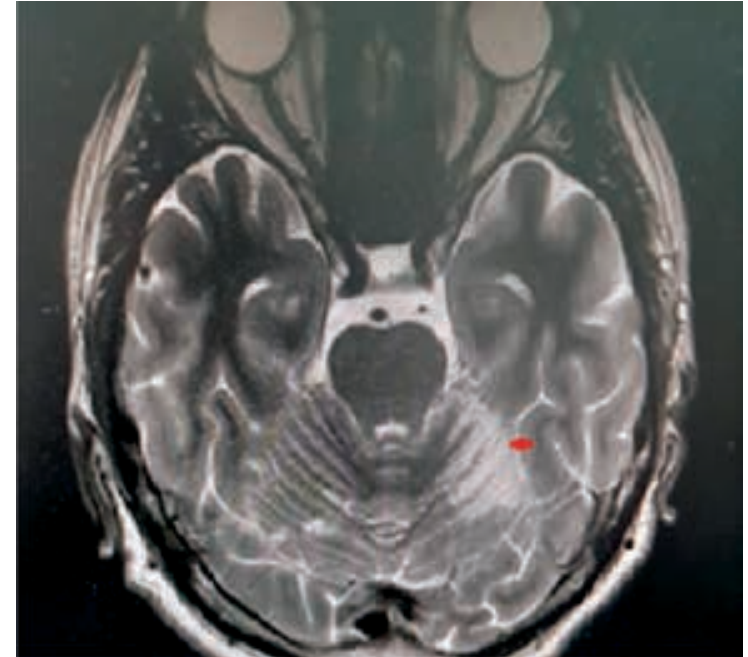

FIGURE 3. Brain magnetic resonance imaging. Axial section. T2. Hyperintensity in left cerebellar lobe

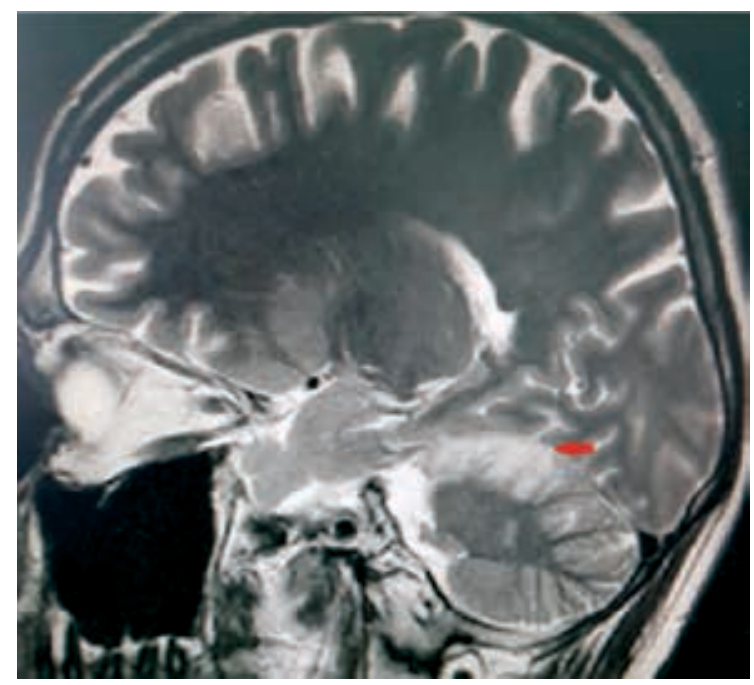

FIGURE 4. Brain magnetic resonance imaging. Sagittal section. T2. Hyperintensity in the upper portion of the cerebellum. Superior cerebellar artery

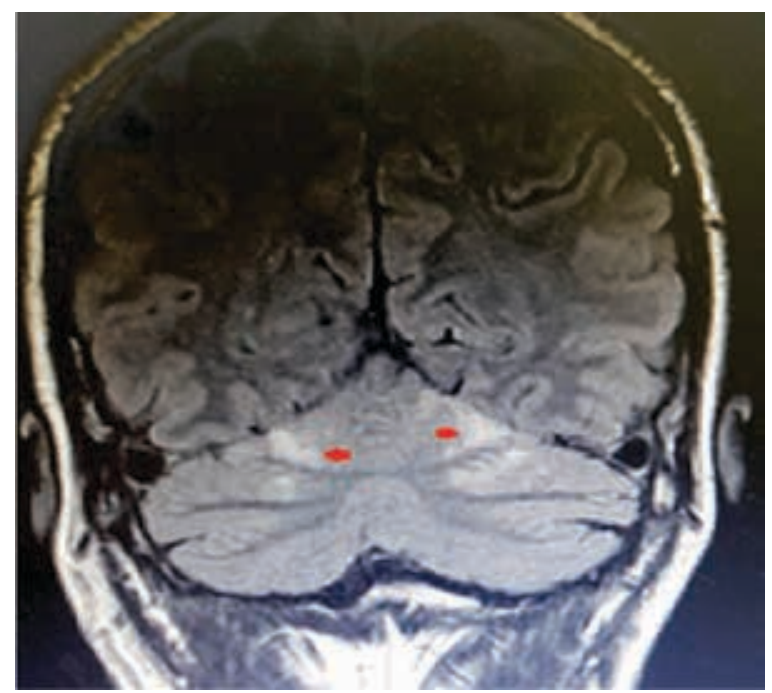

FIGURE 5. Brain magnetic resonance imaging. Coronal section. FLAIR. Hyperintensity signal in bilateral cerebellum hemispheres

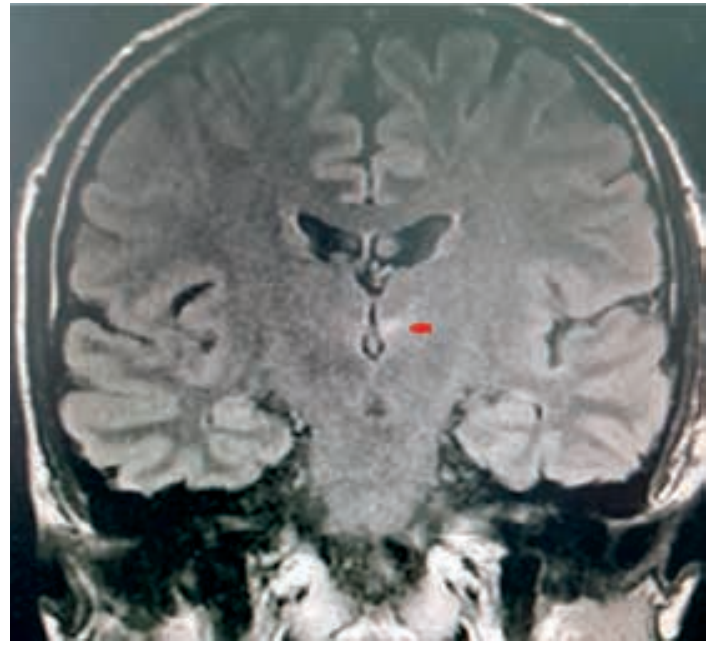

FIGURE 6. Brain magnetic resonance imaging. Coronal section. FLAIR. Minimal hyperintensity left thalamic lesion

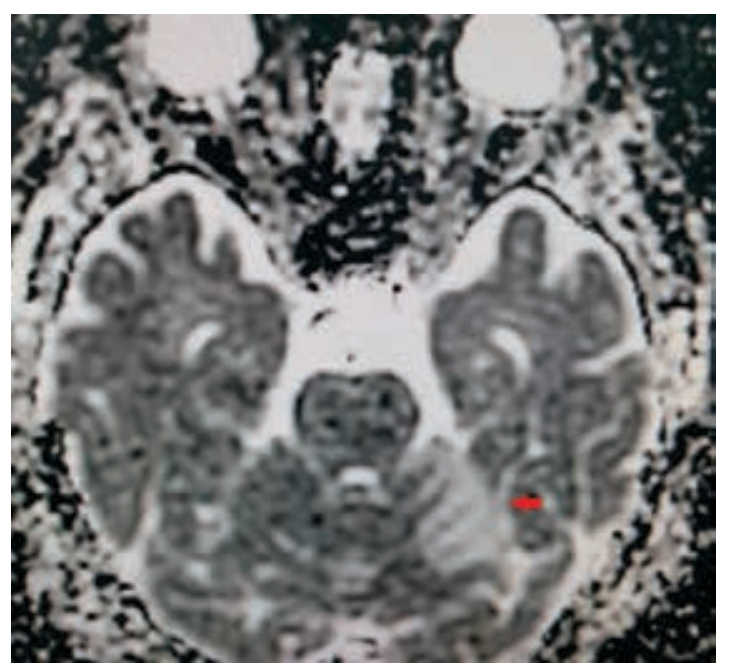

FIGURE 7. Brain magnetic resonance imaging. Axial section. DWI. Minimum diffusion restriction in the left cerebellar lobe

\section{Cardiologic evaluation}

Transthoracic ultrasound (TTE) didn't show structural cardiac modification. Holter ECG in 24 hours didn't describe many important modifications, only a few monomorphic supraventricular extrasystoles considered insignificant.

Transesophageal ultrasound (TEE) reveals a patent foramen ovale with $6 \mathrm{~mm}$ size and an atrial septal aneurysm.

\section{Cardiovascular surgery}

Atrial septal surgery correction was made by implanting the Amplatzer device. After interventional treatment, the patient was treated with double antiaggregant treatment (Aspirin $100 \mathrm{mg}$ /day and Clopidogrel $75 \mathrm{mg} /$ day for 1 to 3 months).

After 3 months, the patient made a transcranial ultrasound with microbbubles contrast agent which 


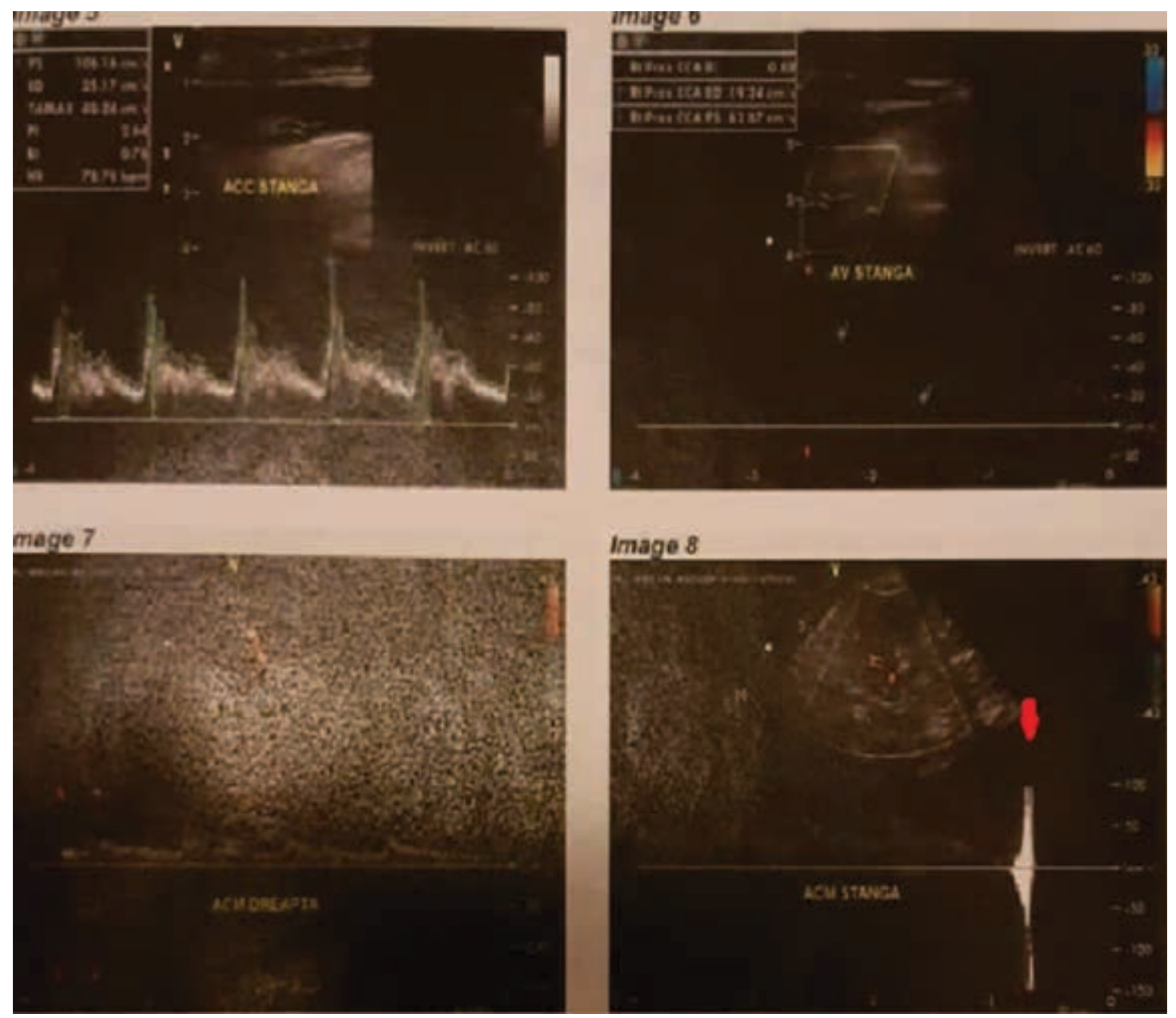

FIGURE 8. High intensity transients (HITS)

revealed high intensity transients-HITs in the middle brain artery (Figure 8). This signal showed that the right-to-left shunt was permeable. In this case, we decided to start anticoagulant therapy with vitamin K inhibitor (Sintrom).

\section{DISCUSSIONS}

Strokes in young people represent $10 \%$ (12). Almost half of those younger don't have a specific cause (cryptogenic stroke) (13). Among the most common causes of stroke in this period are: vertebral and carotid artery dissection, thrombophilia disorders and procoagulant status, paradoxical embolism, vasculitis.

Symptoms suddenly installed like in our case describe the temporal profile very well which is specific to the embolic mechanism. Because, the patient is young, first we considered the posibility of having a vertebral or carotid artery dissection. Brain compiuted tomography with angiography in urgency didn't reveal our suspicion, but we found a right hypoplastic vertebral artery.
The risk of stroke conferred by the presence of atrial fibrillation varies with age, for $1 \%$ for patients under 65 years to $8 \%$ for patients over 65 years. Paroxistic atrial fibrillation is another cause of stroke with embolic mechanism. Different studies showed that this paroxistic atrial fibrillation is more dangerous than chronic cardiac arrhythmias (14). In this case, Holter ECG for 24 hours was normal, but this result does not rule out the possibility of such a rhythm disorder. Paroxistic and asymptomatic atrial fibrillation is difficult to find when a short recording was used, but not in the long recordings (loop recorder). These long recordings increase the rate of fibrillation detection with $3 \%$ to $15 \%$ (14). The hyperthyroidism can determine episodes of atrial fibrillation. In this case, we thought that it was useful to take thyroid hormones (ATPO, ATG, FT4, TSH) which were normal.

A young patient with neurological signs can produce many differential diagnosis issues like multiple sclerosis, migraines which can be excluded 
very easily just with a good anamnestic evaluation. In this case, multiple sclerosis was excluded based on the temporal profile of the onset of the deficit, which in the case of multiple sclerosis, the deficit sets in much more slowly. Migraine was excluded because the patient didn't have specific symptoms.

Vasculitis is another cause of stroke in young patients. Immunological markers (AAN, CIC, p-ANCA, Atc anti RNP/SM/RO CCP) denied this possibility.

Inherited thrombophilias can explain a part of strokes in young patients (15). Thrombophilia disorders are divided into acquired and inherited. Inherited types are far less commonly observed in young adults with ischemic events. This include: protein $\mathrm{S}$, protein $\mathrm{C}$, antithrombin III deficiency, resistance to activated protein $\mathrm{C}$ (factor $\mathrm{V}$ Leiden mutation-FVL). Acquired thrombophilia disorders are more common. They include: the antiphospholipid syndrome associated with lupus anticoagulant and anticardiolipin antibodies (31). For many years, scientists tried to divide patients with stroke and the probability of thrombophilia by age categories so that testing would be more effective and low cost. This category includes young patients under 45 years old $(16,17)$, patients with cryptogenic mechanism $(18,19)$, patients with history of thrombophilia and patent foramen ovale $(20,21)$. In our case, MTHFRc and heterozygous V factor mutation were the only genetic changes. It is not very clear the implication of these mutations in procoagulant status in strokes young patients. Heterozygous $\mathrm{V}$ factor mutation is found in half of the inherited cases and this mutation predisposes to a higher risk of venous thrombosis (31). A retrospective study with 1710 young patients from 2011 to 2015 showed an association of patent foramen ovale with thrombophilic mutations in $28 \%$ of cases. The most frequent mutation was MTHFR which in the absence of folic acid and vitamin B12 deficiency has an uncertain significance. Comparable to the Florez study, the final results didn't show any association between patent foramen ovale and thrombophilic mutations (22). For about ischemic stroke we have only one prospective observation (patients with cryptogenic stroke with and without thrombophilia) where the use of anticoagulation did not affect outcomes. For asymptomatic patient oral anticoagulation is not recommended. But for symptomatic patient it is possible to use the same treatment that is used in venous thromboembolism (treatment with oral anticoagulation) (31).

In our case, brain magnetic resonance imaging reveals many ischemic lesions in the vertebral-basilar territory (bilateral superior cerebellar artery areas). The imaging aspect, the sudden onset, the physical effort, young age, hydrostatic varicose veins were very important in the orientation towards the possibility of paradoxical embolization. In 1998, Lechat and his collaborators were the first who showed the paradoxical embolic issues. They suggested that due to the increased prevalence of clinical venous thrombosis, paradoxical embolism in patent foramen ovale can be responsible for ischemic strokes. Other studies showed that $40 \%$ of ischemic young patients with unknown embolic sources have patent foramen ovale $(2,3,4)$. Patients with ESUS have clinical-radiological characteristics which depend on the following causes: aortic atheromatosis, atrial fibrillation, patent foramen ovale. These young people with PFO had an involvement of the posterior circulation compared to patients with atrial fibrillation. A single photon emission computed tomography study reveals that, in the Valsalva maneuver, the blood flow in posterior circulation was higher than in the previous circulation. This thing can be an explanation to the posterior ischemic events in patients with PFO $(5,6,7)$. Stroke phenotypes differ in patients with PFO depending on the size of the septal defect. This determines patterns of lesions on the diffusion sequence (DWI). Patients with big shunt were associeted with small lesions and $40 \%$ of patients with small shunt had big ischemic lesions on the MRI. It was suggested that another mechanism (together with paradoxical events) can be responsible for these ischemic lesions (9).

For patent foramen ovale diagnosis and the presence of the right-to- left shunt, the transesophageal ultrasound (TEE) is the gold standard, but this investigation can be replaced with transcranial ultrasound (TCD) with microbbubles contrast agent. Of course, the least investigation can't be useful to show the septal defect morphology. American reports from 2004 describe that TEE and TCD have the same sensitivity and specificity in right-to-left shunt detection (23). In our case, TEE revealed a patent foramen ovale with $6 \mathrm{~mm}$ 
size and an atrial septal aneurysm (ASA). A meta analysis of studies published up to 2001 (Mas and his collaborators) showed that the PFO with ASA was associated with ischemic events in patients under 55 years old. It is interesting how patients with just PFO have had a $2 \%$ risk to have a secundar ischemic event, $15 \%$ in patients with $\mathrm{PFO}$ with ASA and $4 \%$ in those people who didn't have any septal defect (24). Without paradoxical mechanism, it is obvious that the high PFO (size $>2 \mathrm{~mm}$ ) and ASA were considered responsible for left atrial dysfunction. This makes a similar atrial fibrillation physiopathology which would lead to embolic events, but reduced ones after correction of the defect (25). These show the importance not only of the surgical correction but also of anticoagulation treatment in the prevention of secondary stroke.

Because not all cases with $\mathrm{PFO}$ can be responsible for stroke events (1/3 of PFO cases are incidental), it was necessary to calculate a risk score for paradoxical embolism. This score is very important because an interventional correction could expose the patient to many risks (26). RoPE (table 1 ) is a risk score which is calculated for the patient with PFO and this describes the ability of a PFO to be responsible for the ischemic event. This risk score uses patient clinical profile, types of ischemic lesion, anatomical features of PFO and conditions that may predispose to paradoxical embolism (physical effort). Our patient had 8 points which meant possibility to have a high paradoxical embolism risk. This score demonstrated association between PFO and the ischemic events. A score of 10 points is calculated based on a number of variables: young adults, ischemic stroke on the MRI/CT, absence of diabetes, absence of high blood pressure and other previous strokes.

Over times, there have been many studies which evaluated the score validity. A small study showed that a RoPE score over 7 points indicates that the PFO is associeted with ischemic stroke (27). In another study, the right-to-left shunt prevalence was $50-56 \%$ for patients with RoPE score under 7 points and $79 \%$ for patients with RoPE score over 7 points (28).

In therapeutic management the main goal is the prevention of secondary stroke. There are two prevention possibilities: either the pharmaceutical treatment (anticoagulant/ antiplatelet) or surgical correction. Anticoagulant treatment should be
ABLE 1. RoPE risk score

\begin{tabular}{|c|c|}
\hline Age & Points \\
\hline $18-29$ & 5 \\
\hline $30-39$ & 4 \\
\hline $40-49$ & 3 \\
\hline $50-59$ & 2 \\
\hline $60-69$ & 1 \\
\hline$>70$ & 0 \\
\hline Features & 1 \\
\hline Without high blood pressure & 1 \\
\hline Without diabetes & 1 \\
\hline Without stroke history & 1 \\
\hline Non-smoker & 1 \\
\hline Cortical lesions & $0-10$ \\
\hline Score &
\end{tabular}

much better than antiplatelet treatment in the stroke prevention, because anticoagulation prevents thromboses better. In most studies, the treatment was done with vitamin $\mathrm{K}$ inhibitors (29). Over times, there have been many cases which evaluated effectivness of interventional treatment. Three randomized trial (2012 and 2013) like CLOSURE I, RESPECT, PC-trial didn't demonstrate superiority of surgical treatment of secondary prevention. Recently, a long evaluation of RESPECT study and another three big trials showed decrease of the risk of recuret ischemic stroke. A systematic review and a meta-analysis of controlled, randomized studies (RCP 1990-2018) which compared surgical treatment (closing the PFO) with antitrombotic therapy, revealed that surgical treatment is better than pharmaceutical treatment in secondary stroke prevention. Patients with ASA or right-to-left shunt can benefit more from the surgical treatment (10). Most evidence of PFO closure was obtained with the Amplatzer device which associated with a completely higher closure and a low secondary atrial fibrillation risk (30). Complete endothelialisation lasts about 5 years. So, double antiplatelet therapy is recommanded for 1-6 months after interventional treatment and antiplatelet monotherapy for 5 years (30). There are many complications after interventional treatment such as: residual shunt, atrial arrhythmias, device thrombosis, endocarditis and erosions.

Because the patient had 8 points (RoPE score), an high embolic risk, after cardiovascular surgery evaluation, the opportunity for surgical treatment was evalueted. Big PFO with associated aneurysm 
respond very well to interventional treatment and the majority no longer had any event. In this case, occlusion of the septal defect was practiced by implantation of the Amplatzer device.

After three months, the patient made a transcranial ultrasound with microbbubles contrast agent which reveals high intensity transients-HITs in the middle brain artery.

An observational study with 80 patients (PFO with one or more ischemic events) who benefited from surgical therapy presented paradoxical embolism in a very small number ( 8 events). It was found that the risk of recurrence was highest in the first year, after the percutaneous closure of the PFO. However, gender and age didn't influence the risk of recurrent thromboembolism. During 5 years of monitoring there was no statistically significant difference in the annual risk of transient ischemic attack, stroke, systemic embolism between patients with $\mathrm{PFO}$ and patients with PFO with ASA. When reevaluated by color flow imaging or bubble contrast injection, after Valsalva maneuver, almost $27 \%$ of the patients had residual right-to-left cardiac shunting. This shunt was a predictor of recurrent thromboembolic events. So, this study demonstrated that the percutaneous closure of the PFO can have a high success rate (>95\%), but with a small complication risk $(10 \%)$ (11).

Since the patient had a septal aneurysm, thrombophilia mutations and the right-to-left shunt was still permeable, we considered that the risk of ischemic stroke was high. Instead of the continuation of the antiplatelet monotherapy, according to the guidelines, the possibility of initiating anticoagulant treatment was re-evaluated. Because the patient had heterozygous $\mathrm{V}$ factor mutation (which is a major risk factor in venous thrombosis), the presence of atrial septal permeability and the presence of septal aneurysm which can cause the formation of small thrombus, anticoagulant therapy was started. Because we have a small number of cases with postsurgical permeable right-to-left shunt mentioned in literature and due to the impossibility of conducting a study that would generate concrete results, the antithrombotic efficiency treatment and prognosis are not very clear. In this case, the necessity of future studies which can clarity treatment issues and future stroke risk in this age group, is clear.

\section{CONCLUSIONS}

In cryptogenic stroke, PFO is a cause which has to be considered when the patient is young ( $<45$ years), with sudden symtoms, after physical effort and cortical ischemic lesions, especially in the vertebrobasilar territory.

Thrombophilia mutations are common. MTHFR mutation has frequently been associated with PFO. Heterozygous $\mathrm{V}$ factor mutation is one of the major risk factors for venous thrombosis.

The more common ischemic events in the basilar vertebro territory in patients with FOP are due to a higher rate of blood flow at this level than in the previous arterial territory during exercise.

RoPE score is a useful score to detect the cases where PFO is responsible for ischemic stroke.

Transesophageal ultrasound is gold standard in PFO diagnosis. Transcranial ultrasound with microbbubles contrast agent has the same sensitivity and specificity in detecting right-to-left shunt.

Patent foramen ovale ( $>2 \mathrm{~mm}$ ) with septal aneurysm is associated with ischemic events.

Almost quarter of the patients can have residual right-to-left cardiac shunting after interventional treatment.

Although standard treatment (surgical terapy) has an excellent therapeutic response, because it can reduce the risk of a new stroke to almost zero, there are rare situations with residual right-to-left cardiac shunting which can pose therapeutic management problems.

\section{REFERENCES}

1. Hart RG, Diener HC, Coutts SB, Easton JD, Granger CB, O'Donnell MJ, Sacco RL, Connolly SJ; Cryptogenic Stroke/ESUS International Working Group. Embolic strokes of undetermined source: the case for a new clinical construct. Lancet Neurol. 2014 Apr;13(4):429-38.

2. Lechat P, Mas JL, Lascault G, Loron P, Theard M, Klimczac M, Drobinski G, Thomas D, Grosgogeat Y. Prevalence of patent

foramen ovale in patients with stroke. N Engl J Med. 1988 May 5;318(18):1148-52.

3. Hagen PT, Scholz DG, Edwards WD. Incidence and size of patent foramen ovale during the first 10 decades of life: an autopsy study of 965 normal hearts. Mayo Clin Proc. 1984 Jan;59(1):17-20. 
4. Overell JR, Bone I, Lees KR. Interatrial septal abnormalities and stroke: a meta-analysis of case-control studies. Neurology. 2000 Oct 24;55(8):1172-9.

5. Jauss M, Zanette E. Detection of right-to-left shunt with ultrasound contrast agent and transcranial Doppler sonography. Cerebrovasc Dis. 2000 Nov-Dec;10(6):490-6.

6. Bang OY, Ovbiagele B, Kim JS. Evaluation of cryptogenic stroke with advanced diagnostic techniques. Stroke. 2014 Apr; 45(4):1186-94.

7. Kim BJ, Sohn H, Sun BJ, Song JK, Kang DW, Kim JS, Kwon SU. Imaging characteristics of ischemic strokes related to patent foramen ovale. Stroke. 2013 Dec;44(12):3350-6.

8. Hayashida K, Fukuchi K, Inubushi M, Fukushima K, Imakita S, Kimura K. Embolic distribution through patent foramen ovale demonstrated by (99m)Tc-MAA brain SPECT after Valsalva radionuclide venography. J Nucl Med. 2001 Jun;42(6):859-63.

9. Kim JW, Kim SJ, Yoon CW, Park CH, Kang KW, Kim SK, Kim YH, Bang OY. Association between the amount of right-to-left shunt and infarct patterns in patients with cryptogenic embolic stroke: a transcranial Doppler study. Int J Stroke. 2013 Dec;8(8):657-62.

10. Turc G, Calvet D, Guérin P, Sroussi M, Chatellier G, Mas JL; CLOSE Investigators. Closure, Anticoagulation, or Antiplatelet Therapy for Cryptogenic Stroke With Patent Foramen Ovale: Systematic Review of Randomized Trials, Sequential Meta-Analysis, and New Insights From the CLOSE Study. J Am Heart Assoc. 2018 Jun 17.

11. Windecker S, Wahl A, Chatterjee T, Garachemani A, Eberli FR, Seiler C, Meier B. Percutaneous closure of patent foramen ovale in patients with paradoxical embolism: long-term risk of recurrent thromboembolic events. Circulation. 2000 Feb 29;101(8):893-8.

12. George MG, Tong X, Kuklina EV, Labarthe DR. Trends in stroke hospitalizations and associated risk factors among children and young adults, 1995-2008. Ann Neurol. 2011 Nov;70(5):713-21.

13. Sultan S, Elkind MS. The growing problem of stroke among young adults. Curr Cardiol Rep. 2013 Dec;15(12):421.

14. Allan H. Rapper. MD, Martin A.Samuels MD, Joshua P. Klein. MD. $\mathrm{PhD}$. Adams and Victor's Principles of Neurology 10th Edition. pag 784 .

15. Ji R, Schwamm LH, Pervez MA, Singhal AB. Ischemic stroke and transient ischemic attack in young adults: risk factors, diagnostic yield, neuroimaging, and thrombolysis. JAMA Neurol. 2013 Jan;70(1):51-7.

16. De Stefano V, Chiusolo P, Paciaroni K, Casorelli I, Rossi E, Molinari M, Servidei S, Tonali PA, Leone G. Prothrombin G20210A mutant genotype is a risk factor for cerebrovascular ischemic disease in young patients. Blood. 1998 May 15;91(10):3562-5.

17. Hamedani AG, Cole JW, Mitchell BD, Kittner SJ. Meta-analysis of factor $\mathrm{V}$ Leiden and ischemic stroke in young adults: the importance of case ascertainment. Stroke. 2010 Aug;41(8):1599-603.

18. Aznar J, Mira Y, Vayá A, Corella D, Ferrando F, Villa P, Estellés A. Factor V Leiden and prothrombin G20210A mutations in young adults with cryptogenic ischemic stroke. Thromb Haemost. 2004 May;91(5):1031-4.

19. Belvís R, Santamaría A, Martí-Fàbregas J, Cocho D, Borrell M, Fontcuberta J, Martí-Vilalta JL. Diagnostic yield of prothrombotic state studies in cryptogenic stroke. Acta Neurol Scand. 2006 Oct;114(4):250-3.

20. Nabavi DG, Junker R, Wolff E, Lüdemann P, Doherty C, Evers S, Droste DW, Kessler C, Assmann G, Ringelstein EB. Prevalence of factor $V$ Leiden mutation in young adults with cerebral ischaemia: a case-control study on 225 patients. J Neurol. 1998 Oct; 245(10):653-8.

21. Florez JC, Ay H, Van Cott EM, Buonanno FS. Patent foramen ovale and hypercoagulability as combined risk factors for stroke. J Stroke Cerebrovasc Dis. 2003 May-Jun;12(3):114-8.

22. Omran SS, Lerario MP, Gialdini G, Merkler AE, Moya A, Chen ML, Kamel H, DeSancho M, Navi BB. Clinical Impact of Thrombophilia Screening in Young Adults with Ischemic Stroke. J Stroke Cerebrovasc Dis. 2019 Apr;28(4):882-889.

23. Walter S, Kostopoulos P, Haass A, Keller I, Lesmeister M, Schlechtriemen T, Roth C, Papanagiotou P, Grunwald I, Schumacher H, Helwig S, Viera J, Körner H, Alexandrou M, Yilmaz U, Ziegler K, Schmidt K, Dabew R, Kubulus D, Liu Y, Volk T, Kronfeld K, Ruckes C, Bertsch T, Reith W, Fassbender K. Diagnosis and treatment of patients with stroke in a mobile stroke unit versus in hospital: a randomised controlled trial. Lancet Neurol. 2012 May;11(5):397-404.

24. Fassbender K, Balucani C, Walter S, Levine SR, Haass A, Grotta J. Streamlining of prehospital stroke management: the golden hour. Lancet Neurol. 2013 Jun;12(6):585-96.

25. Rigatelli G, Aggio S, Cardaioli P, Braggion G, Giordan M, Dell'avvocata F, Chinaglia M, Rigatelli G, Roncon L, Chen JP. Left atrial dysfunction in patients with patent foramen ovale and atrial septal aneurysm: an alternative concurrent mechanism for arterial embolism? JACC Cardiovasc Interv. 2009 Jul;2(7):655-62.

26. Kent DM, Ruthazer R, Weimar C, Mas JL, Serena J, Homma S, Di Angelantonio E, Di Tullio MR, Lutz JS, Elkind MS, Griffith J, Jaigobin C, Mattle HP, Michel P, Mono ML, Nedeltchev K, Papetti F, Thaler $D E$. An index to identify stroke-related vs incidental patent foramen ovale in cryptogenic stroke. Neurology. 2013 Aug 13;81(7):619-25.

27. Prefasi D, Martínez-Sánchez P, Fuentes B, Díez-Tejedor E. The utility of the RoPE score in cryptogenic stroke patients $\leq 50$ years in predicting a stroke-related patent foramen ovale. Int J Stroke. 2016 Jan;11(1):NP7-8.

28. Wessler BS, Kent DM, Thaler DE, Ruthazer R, Lutz JS, Serena J. The RoPE Score and Right-to-Left Shunt Severity by Transcranial Doppler in the CODICIA Study. Cerebrovasc Dis. 2015;40(1-2):52-8.

29. Saver JL, Mattle HP, Thaler D. Patent Foramen Ovale Closure Versus Medical Therapy for Cryptogenic Ischemic Stroke: A Topical Review. Stroke. 2018 Jun;49(6):1541-1548.

30. Pristipino C, Sievert H, D'Ascenzo F, Mas JL, Meier B, Scacciatella P, Hildick-Smith D, Gaita F, Toni D, Kyrle P, Thomson J, Derumeaux G, Onorato E, Sibbing D, Germonpré P, Berti S, Chessa M, Bedogni F, Dudek D, Hornung M, Zamorano J; European Association of Percutaneous Cardiovascular Interventions (EAPCI); European Stroke Organisation (ESO); European Heart Rhythm Association (EHRA); European Association for Cardiovascular Imaging (EACVI); Association for European Paediatric and Congenital Cardiology (AEPC); ESC Working group on GUCH; ESC Working group on Thrombosis; European Haematological Society (EHA). European position paper on the management of patients with patent foramen ovale. General approach and left circulation thromboembolism. Eurolntervention. 2019 Jan 20;14(13):1389-1402.

31. Ng KW, Loh PK, Sharma VK. Role of investigating thrombophilic disorders in young stroke. Stroke Res Treat. $2011 \mathrm{Feb}$ 8;2011:670138.

Conflict of interest: none declared

Financial support: none declared 